\title{
Esophageal laceration after percutaneous endoscopic gastrostomy tube removal attempt: alternative route of extraction
}

Removal of a percutaneous endoscopic gastrostomy (PEG) tube is recommended after patients resume oral intake [1]. There are three main techniques described to remove a PEG tube: 1) external traction at the skin level; 2) the cut and push technique; and 3) retrograde esophageal exteriorization under endoscopic control [1 - 3]. The choice depends mainly on endoscopist preference and local guidelines. Several risk factors for tube deterioration have been described, such as heat and fungal colonization [3, 4].

We report the case of an 83-year-old woman with a PEG tube inserted 2 years previously for dysphagia in the context of Parkinson's disease. After PEG placement, the patient did not attend any of the scheduled appointments. After 2 years, the patient's family contacted our
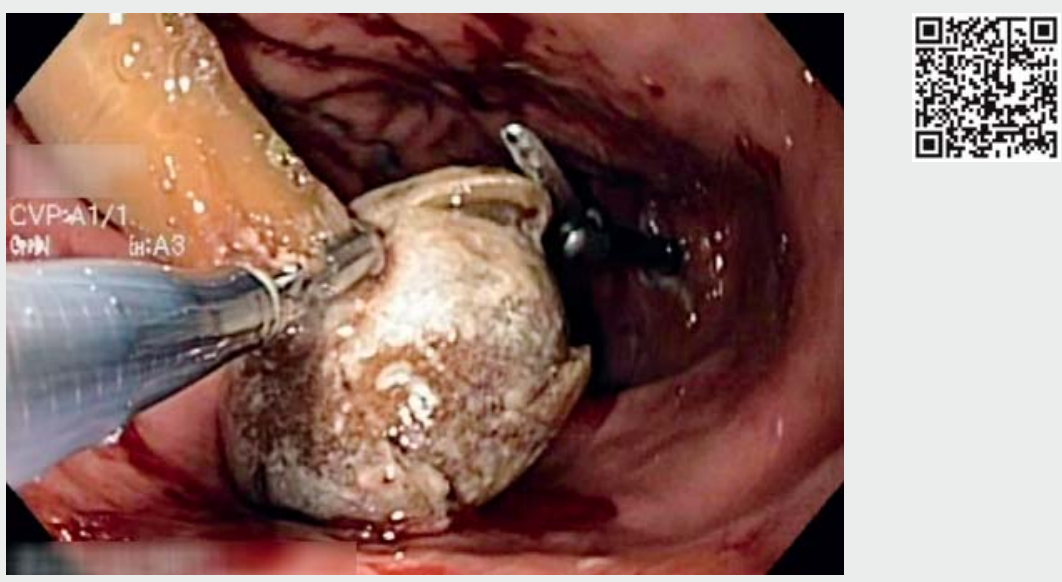

Video 1 Use of laparoscopic scissors inserted through a gastrostomy tract to perform multiple radial incisions in a stony bumper in order to facilitate its collapse when extracting it through the stoma.
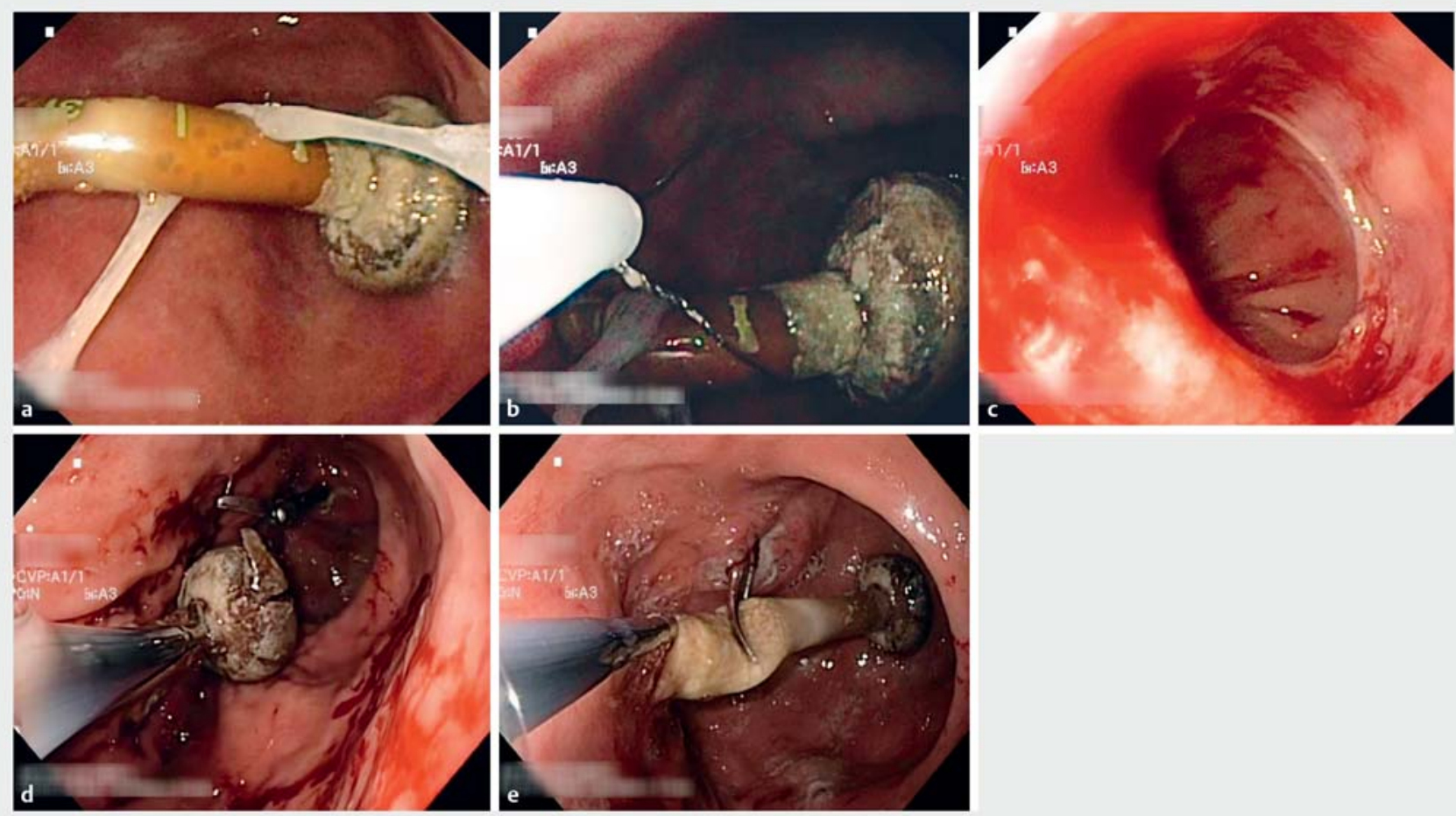

- Fig. 1 Endoscopic view of percutaneous endoscopic gastrostomy (PEG) tube removal. a The PEG tube in place. $\mathbf{b}$ Ensnarement of the PEG tube after cutting it externally. $\mathbf{c}$ Esophageal laceration at the lower esophageal sphincter. $\mathbf{d}$ Multiple radial incisions in the bumper using a laparoscopic scissor inserted through the gastrostomy tract. e Forceps inserted through the stoma in order to pull out the PEG tube. 
Department to inform us that the patient had returned to oral intake after a year and the feeding tube was redundant. Initially, we tried to remove the tube endoscopically through the mouth ( $\vee$ Video 1 ) as is standard practice in our department ( Fig.1a,b). During the procedure, an esophageal laceration occurred at the lower esophageal sphincter owing to stiffness of the bumper ( $\triangleright$ Fig. 1 c). Given the risks associated with attempting to remove the PEG tube through the esophagus, we decided to remove it through the gastrostomy tract.

Given the rigidity of the bumper, we performed multiple radial incisions on the bumper using a laparoscopic scissor inserted through the gastrostomy tract, to facilitate its collapse when extracting it through the stoma ( $\triangleright$ Fig. 1 d,e).

At the end of the procedure, we confirmed the PEG tube was rigid, with a marked decrease in its elasticity. Culture of the PEG material showed fungal colonization (hyphae growth).

Endoscopists who choose the oral route to remove PEG tubes should be aware that the original properties of the tube can be modified over time, namely its elasticity, which can render the oral route hazardous for removal. This case report demonstrates an alternative route for PEG removal in this setting.

Endoscopy_UCTN_Code_CPL_1AH_2AI
Acknowledgments

The authors gratefully acknowledge the generous assistance of all of the endoscopy unit staff.

\section{Competing interests}

None

\section{The authors}

João Fernandes ${ }^{1}$, Rui Ramos ${ }^{1}$, Célia Vicente ${ }^{1}$, Tobias Teles $^{2}$, Jorge Canena ${ }^{3}$, Luís Lopes ${ }^{4,5,6}$, Carlos Casteleiro ${ }^{1}$

1 Gastroenterology Department, Centro Hospitalar Universitário Cova da Beira EPE, Covilhã, Portugal

2 Surgery Department, Centro Hospitalar Universitário Cova da Beira EPE, Covilhã, Portugal

3 Nova Medical School/FCML, Universidade Nova de Lisboa, Lisboa, Portugal

4 Gastroenterology Department, Hospital Santa Luzia, Viana do Castelo, Portugal

5 Life and Health Sciences Research Institute (ICVS), School of Medicine, University of Minho, Braga, Portugal

6 ICVS/3B's - PT Government Associate Laboratory, Braga/Guimarães, Portugal

\section{Corresponding author}

\section{João Fernandes, MD}

Department of Gastroenterology, Hospital de Pêro da Covilhã, Centro Hospitalar Universitário Cova da Beira EPE, Quinta do Alvito, Covilhã 6200-251, Portugal Fax: +351-275-751057

Jotillfernandes@hotmail.com

\section{References}

[1] Rahnemai-Azar AA, Rahnemaiazar AA, Naghshizadian $R$ et al. Percutaneous endoscopic gastrostomy: indications, technique, complications and management. World J Gastroenterol 2014; 20: 7739-7751

[2] Löser C, Aschl G, Hébuterne X et al. ESPEN guidelines on artificial enteral nutrition percutaneous endoscopic gastrostomy (PEG). Clin Nutr 2005; 24: 848-861

[3] Heuschkel RB, Gottrand F, Devarajan K et al. ESPGHAN position paper on management of percutaneous endoscopic gastrostomy in children and adolescents. J Pediatr Gastroenterol Nutr 2015; 60: 131-141

[4] Sartori S, Trevisani L, Nielsen I et al. Longevity of silicone and polyurethane catheters in long-term enteral feeding via percutaneous endoscopic gastrostomy. Aliment Pharmacol Ther 2003; 17: $853-856$

\section{Bibliography}

DOI https://doi.org/10.1055/a-0896-2430

Published online: 13.5.2019

Endoscopy 2019; 51: E274-E275

(c) Georg Thieme Verlag KG

Stuttgart · New York

ISSN 0013-726X

\section{ENDOSCOPY E-VIDEOS}

https://eref.thieme.de/e-videos

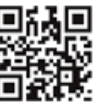

Endoscopy E-Videos is a free access online section, reporting on interesting cases and new

techniques in gastroenterological endoscopy. All papers include a high quality video and all contributions are freely accessible online.

This section has its own submission website at https://mc.manuscriptcentral.com/e-videos 